\title{
High-Throughput Sequencing of Putative Novel microRNAs in Rhesus Monkey Peripheral Blood Mononuclear Cells following EV71 and CA16 Infection
}

\author{
Jie Song Xi Jiang Yajie Hu Hui Li Xuemei Zhang Jingwen Xu Weiyu Li \\ Xuelin Zheng Shaozhong Dong \\ Yunnan Key Laboratory of Vaccine Research and Development on Severe Infectious Disease, Institute of \\ Medical Biology, Chinese Academy of Medical Science and Peking Union Medical College, Kunming, China
}

\section{Keywords \\ Hand, foot, and mouth disease - Enterovirus 71 . \\ Coxsackievirus A16 $\cdot$ microRNAs $\cdot$ High-throughput \\ sequencing $\cdot$ Peripheral blood mononuclear cells}

\begin{abstract}
Objectives: Enterovirus 71 (EV71) and Coxsackievirus A16 (CA16) remain the major pathogens in hand, foot, and mouth disease (HFMD) cases, but the mechanisms of the different pathogeneses that follow EV71 and CA16 infection remain largely unknown. Methods: Herein, we utilized microRNA (miRNA) deep sequencing to investigate the roles of novel differentially expressed miRNAs in peripheral blood mononuclear cells (PBMCs) infected with EV71 and CA16. Results: The results identified 13 novel differentially expressed miRNAs in each group. Additionally, the target genes were predicted by the miRanda and RNAhybrid programs, and a total of 2,501 targets were found in the two databases. Then, Gene Ontology (GO) and Kyoto Encyclopedia of Genes and Genomes (KEGG) enrichment analyses revealed that these targets were mainly involved in cell development and were
\end{abstract}

\section{KARGER}

(c) 2018 S. Karger AG, Basel

E-Mail karger@karger.com

www.karger.com/int associated with nervous system development, system development, multicellular organism development, the Wnt signaling pathway, the PDGF signaling pathway, and the EGF receptor signaling pathway. Finally, a coexpression regulatory network was built with the key targets to further extrapolate the functional interactions of the targets and their coexpressed genes. Conclusion: Our results not only revealed potential biomarkers or targets for the diagnosis and treatment of HFMD, but also provided new insights to explore the mechanisms of EV71 and CA16 pathogenesis.

(c) 2018 S. Karger AG, Basel

\section{Introduction}

Enterovirus 71 (EV71) and Coxsackievirus A16 (CA16) are two of the most common etiologic agents for hand, foot, and mouth disease (HFMD). HFMD, which is mostly seen in infants and young children under the age

J.S. and X.J. contributed equally to this work.
Shaozhong Dong, $\mathrm{PhD}$

Institute of Medical Biology, Chinese Academy of Medical Sciences and Peking Union Medical College, 935 Jiaoling Road

Kunming, Yunnan 650118 (China)

E-Mail dszimbcams@163.com 
of 5 years, is characterized by a maculopapular rash or blisters on the hands, feet, groin, and buttocks, and can also include painful ulcerative lesions of the mouth [1-3]. Although most cases are mild and self-limiting with an average duration of 7 days, approximately $1 \%$ of cases can rapidly develop severe and even life-threatening complications, including aseptic meningitis, cerebellar ataxia, poliomyelitis-like paralysis, acute brainstem encephalitis, cardiopulmonary failure, and fulminant neurogenic pulmonary edema associated with high mortality [4]. An epidemiological survey revealed that since the 1990s, the HFMD epidemic has mainly affected the Asia-Pacific region; it has thus become a serious threat to public health across this region [5]. Fortunately, three EV71 vaccines from manufacturers at the Institute of Medical Biology at the Chinese Academy of Medical Science (CAMS), Sinovac Biotech Ltd. (Sinovac), and Beijing Vigoo Biological Co. Ltd. (Vigoo), were recently licensed by the China Food and Drug Administration (CFDA) $[3,6]$. It was widely believed that these vaccines would markedly reduce the incidence of HFMD, particularly the morbidity and mortality of severe HFMD; however, they only protected against the fraction of HFMD cases caused by EV71 and could not effectively control the HFMD epidemic induced by other enteroviruses, including CA16 [7]. However, various genotypes of enteroviruses have often been found to alternate between circulation and cocirculation, resulting in HFMD epidemics in children [8, 9]. Thus, the need to develop a multivalent vaccine against multiple enteroviruses is of high urgency.

microRNAs (miRNAs), an abundant group of endogenous, highly conserved noncoding single-stranded RNAs (22 nt in length), suppress approximately $60 \%$ of human genes by binding to the $3^{\prime}$ untranslated region (UTR) of target message RNAs (mRNAs), causing target cleavage or translational blockage [10]. They have been detected in many plant and animal species and even in some animal viral RNA genomes, and have been found to be involved in a wide range of biological processes, including apoptosis, cell proliferation, cell development, cell differentiation, and homeostasis [11]. Moreover, a growing body of evidence has demonstrated that miRNAs can play a key role in the regulation of viral replication. For example, the liver-specific miR-122 interacts with the $5^{\prime}$-UTR of the genome of the hepatitis $\mathrm{C}$ virus (HCV) and increases viral replication [12]. The H1N1 influenza A virus-induced miR-323, miR-491, and miR-654 inhibit viral replication by directly binding to the PB1 gene [13]. miR-23b inhibits the replication of EV71 by targeting a conserved EV71 3'-UTR sequence [14]. These findings suggest that cell-encoded miRNAs can directly affect the virus replication cycle, and investigations at the miRNA level could contribute to further understanding of the mechanisms of the interaction between viruses and host cells and provide scientific information for the discovery of novel antiviral agents and strategies.

In our previous study, we reported that EV71 and CA16 infection can change the expression patterns of miRNAs in various cells, such as human bronchial epithelial (16HBE) cells and human umbilical vein endothelial cells (HUVECs), which are likely to play a role in viral pathogenesis $[1,15]$. Hence, in this study, we aimed to further analyze the functions of novel differentially expressed miRNAs in peripheral blood mononuclear cells (PBMCs) of rhesus monkeys following EV71 and CA16 infection, and to elucidate the possible mechanisms leading to the different outcomes caused by EV71 and CA16 infection.

\section{Materials and Methods}

\section{Virus Infection and Cell Culture}

PBMCs were isolated from EDTA-anticoagulated whole blood samples from healthy rhesus monkeys with no known infections or diseases by Ficoll-Hypaque (TBD Sciences, China) gradient centrifugation according to the manufacturer's instructions. Then, PBMCs were plated onto 6-well plates at a density of $2 \times 10^{5}$ cells per well and were grown in complete culture medium containing RPMI-1640 (Gibco, USA) supplemented with 10\% fetal bovine serum (FBS, Gibco, USA), 2 mmol/L L-glutamine, $100 \mathrm{U} / \mathrm{mL}$ penicillin, and $100 \mu \mathrm{g} / \mathrm{mL}$ streptomycin, and maintained in a humidified incubator at $37^{\circ} \mathrm{C}$ with $5 \% \mathrm{CO}_{2}$. The next day, PBMCs were infected with an EV71 strain (subgenotype C4, GenBankEU812515.1) or a CA16-G20 strain (subgenotype B, GenBank JN590244.1) that had originated from the HFMD epidemic in Fuyang, China, in 2008 or in Guangxi, China, in 2010, respectively, at a multiplicity of infection of 1 . The cells were collected at 0,24 , and $48 \mathrm{~h}$ postinfection, resuspended in TRIzol (TIANGEN, China), and stored at $-80^{\circ} \mathrm{C}$ until used. Cells infected with EV71 and CA16 for $0 \mathrm{~h}$ postinfection were used as controls. We defined the following experimental groups: EV71-0 h, EV71-24 h, EV71-48 h, CA16-0 h, CA16-24 h, and CA16-48 h. Additionally, the EV71-0 h and CA16-0 h groups were designated as controls and used for normalization (the normalization value was set to 1 ).

\section{RNA Isolation and Quality Control}

miRNA was extracted from PBMC samples using a mirVana ${ }^{\mathrm{TM}}$ miRNA Isolation Kit (Invitrogen, Carlsbad, CA, USA) following the manufacturer's instructions. The quality of the isolated RNA was evaluated by the ratio of 18 S/28S RNA using an Agilent 2100 Bioanalyzer (Agilent Technologies, USA) with RNA 6000 Nano LabChips. For further studies, samples with an RNA integrity number $>7.0$ were used. The qualified miRNA from three replicates was pooled as one RNA sample for subsequent library construction and deep sequencing. 
Small RNA Library Construction, High-Throughput

Sequencing, and miRNA-Seq Data Analysis

The RNA samples of each group were used to construct small RNA libraries with a TruSeq Small RNA sample preparation kit (Illumina, USA) according to the manufacturer's instructions. Small RNAs $18-35 \mathrm{nt}$ in length were separated and purified by denaturing polyacrylamide gel electrophoresis. After dephosphorylation and ligation of a pair of adaptors to their $5^{\prime}$ and $3^{\prime}$ ends, the products were reverse-transcribed and amplified by quantitative RT-PCR (RT-qPCR) with gel purification. After they were constructed, the small RNA libraries were sequenced using an Illumina Hi-seq 2000 platform (Illumina Inc., USA). After sequencing, the raw reads were processed into clean reads, the adaptor sequences, poly $(\mathrm{A})$ tails, and inserted tags were removed, the low-quality reads were filtered out (those with ambiguous base nt and quality scores [Qscores] less than 10), and the clean 18-35 nt small RNAs (sRNAs) were mapped using Rfam (http://rfam.janelia.org/) and Piwi-interacting RNA (piRNA) (http://pirnabank.ibab.ac.in/) to remove noncoding RNAs (e.g., ribosomal RNA [rRNA], transfer RNA [tRNA], small nucleolar RNAs [snoRNAs], small nuclear RNA [snRNA]) and repetitive RNAs. The remaining reads from each library were used to identify known miRNAs by alignment to known human miRNA mature sequences obtained from miRBase 19.0 (available online: http://www.mirbase.org/). Finally, potentially novel candidate miRNAs were predicted based on the unannotated sequences using a modified version of the miREvo program. The sequencing data were deposited in the National Center for Biotechnology Information's Gene Expression Omnibus (GEO) database (www.ncbi.nlm.nih.gov/geo/) under accession number GSE85820.

\section{Bioinformatics Analysis}

In our previous study, the known differentially expressed miRNAs were thoroughly analyzed [16]. Hence, in the present study, we mainly implemented bioinformatics analysis for novel miRNAs.

\section{Principal Component Analysis}

Clean data were obtained from the preprocessed miRNA-seq data and then subjected to principal component analysis (PCA) to determine similarities and differences among the groups.

\section{Differential Expression Analysis of Novel miRNAs}

Differentially expressed miRNAs were identified based on the TPM (transcripts per million clean tags) values. We calibrated novel miRNA expression levels using multiple hypothesis tests with a false discovery rate $\leq 0.01$, performed generalized $\chi^{2}$ tests for differential miRNA expression using IDEG6 software (http:// telethon.bio.unipd.it/bioinfo/IDEG6/), and screened the novel miRNAs for those with $p$ values $<0.05$ and fold changes $\geq 2$ or $\leq 0.01$. The novel miRNAs that met these criteria were identified as being differentially expressed.

\section{Clustering Analysis}

A heat map of the novel differentially expressed miRNAs was constructed using the heatmap package in $\mathrm{R}$ (version 3.1.3) [17]. The normalized data were transformed to a $\log _{2}$ scale for visualization purposes.

\section{miRNA Target Prediction}

Target genes of the novel differentially expressed miRNAs were predicted using the miRanda and RNAhybrid programs [18, 19]. We defined putative targets as those with a Score $\geq 150$ and Energy
$<-20$ in miRanda and Energy $<-25$ in RNAhybrid. To increase the reliability of the results, the intersection of the results of these two software programs was ultimately considered to include the genes targeted by novel differentially expressed miRNAs.

\section{GO and KEGG Pathway Enrichment Analysis}

The Gene Ontology (GO) terms and Kyoto Encyclopedia of Genes and Genomes (KEGG) pathways of these novel miRNA targets were annotated using DAVID bioinformatics resources (http:// david.abcc.ncifcrf.gov/). The GO and pathway terms with $p$ values $\leq 0.05$ based on the Benjamini-Hochberg method were defined as significantly enriched GO terms and KEGG pathways [20].

\section{Coexpression Network Analysis}

The GeneMANIA algorithm was applied to construct a network of the overlapped target genes that were involved in both the GO term and KEGG pathway analyses.

Confirmation of Differentially Expressed miRNA and miRNA Target Expression by Quantitative RT-PCR

To validate the sequencing data, we chose 8 differentially expressed miRNAs and their target genes for RT-qPCR analysis. Total RNA was extracted from each sample using TRIzol Reagent (TIANGEN, China) according to the manufacturer's instructions, and the isolated RNA was cleared of contaminating genomic DNA by DNase treatment (Thermo Fisher Scientific, USA). For miRNA analysis, complementary DNA (cDNA) was first synthesized with a poly $(\mathrm{T})$ adapter primer under the following reaction conditions: $60 \mathrm{~min}$ at $37^{\circ} \mathrm{C}, 5 \mathrm{~min}$ at $85^{\circ} \mathrm{C}$, and maintenance at $4^{\circ} \mathrm{C}$. After this was completed, $90 \mu \mathrm{L}$ of $\mathrm{ddH}_{2} \mathrm{O}$ was added to the cDNA for miRNA quantification with an mRQ $3^{\prime}$ primer and miRNA-specific $5^{\prime}$ primers using SYBR Advantage qPCR Premix (Clontech, USA) on an ABI PRISM ${ }^{\circledR} 7500$ Sequence Detection System (Applied Biosystems, USA). The cycle conditions were as follows: $95^{\circ} \mathrm{C}$ for $10 \mathrm{~s}$, followed by 40 cycles of $95^{\circ} \mathrm{C}$ for $10 \mathrm{~s}$ and $60^{\circ} \mathrm{C}$ for $40 \mathrm{~s}$, and then dissociation at $95^{\circ} \mathrm{C}$ for $60 \mathrm{~s}, 55^{\circ} \mathrm{C}$ for $30 \mathrm{~s}$, and $95^{\circ} \mathrm{C}$ for $30 \mathrm{~s}$.

For mRNA analysis, reverse transcription and PCR were performed using a One-Step SYBR ${ }^{\circledR}$ PrimeScript ${ }^{\mathrm{TM}}$ PLUS RT-qPCR Kit (TAKARA, Japan) following the manufacturer's instructions. The RT-qPCR cycling conditions were 1 cycle at $42^{\circ} \mathrm{C}$ for 5 min and 1 cycle at $95^{\circ} \mathrm{C}$ for $10 \mathrm{~s}$, followed by a two-step PCR procedure consisting of $5 \mathrm{~s}$ at $95^{\circ} \mathrm{C}$ and $34 \mathrm{~s}$ at $60^{\circ} \mathrm{C}$ for 40 cycles. Melting curve analysis was performed to verify specific amplification $\left(95^{\circ} \mathrm{C}\right.$ for $60 \mathrm{~s}, 55^{\circ} \mathrm{C}$ for $30 \mathrm{~s}$, and $95^{\circ} \mathrm{C}$ for $30 \mathrm{~s}$ ).

The relative expression levels of miRNAs and mRNAs were calculated by the $2^{-\Delta \Delta C_{t}}$ method and normalized to the expression of universal U6 small nuclear RNA (U6 snRNA) and GAPDH, which were used as endogenous controls for miRNA and mRNA, respectively. For each sample, miRNA and mRNA expression was measured in triplicate. The primers sequences of these genes were designed for this study and are listed in online supplementary Tables S1 and S2 (for all online suppl. material, see www.karger.com/ doi/10.1159/000493798).

\section{Statistical Analysis}

For the sequencing data, the raw reads obtained from each library were normalized to TPM. For RT-qPCR, the data are given as the mean \pm standard error of the mean (SEM). Statistical analysis was performed using SPSS 18.0 (IBM SPSS, USA). Statistical significance was defined as $p<0.05$. 


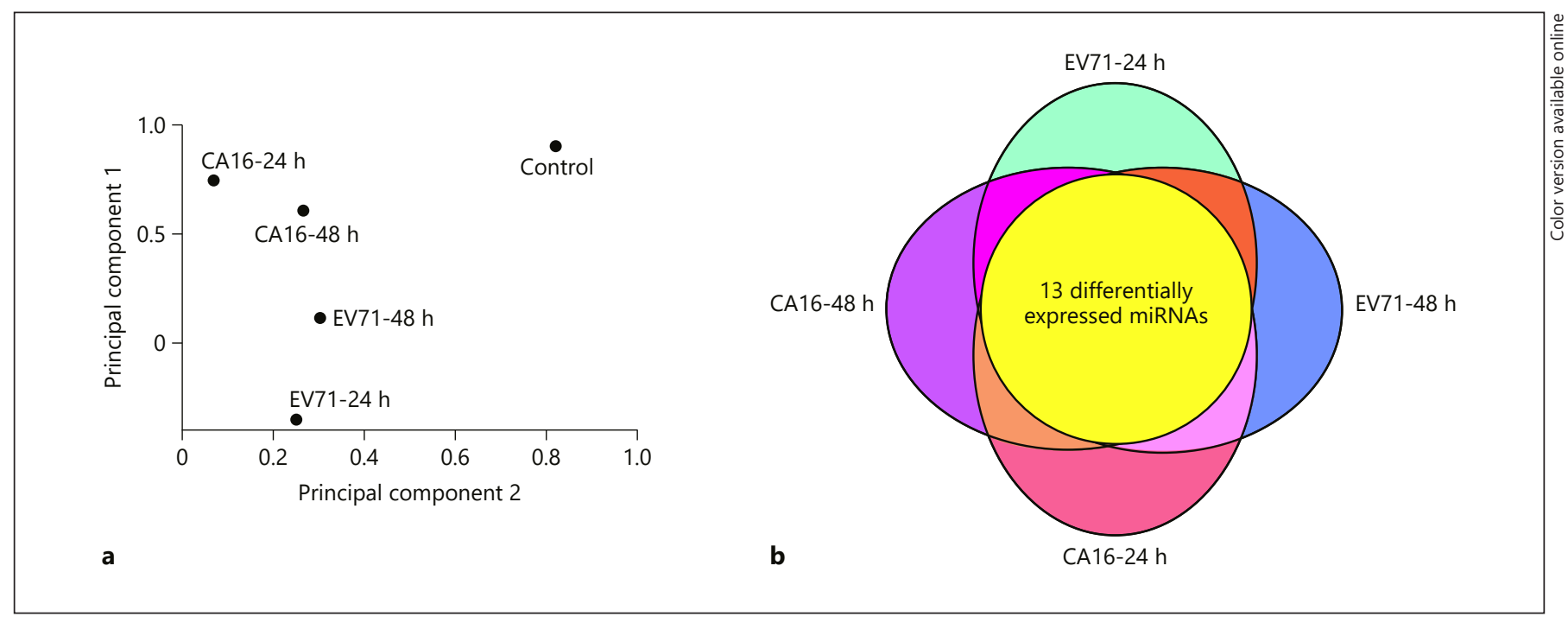

Fig. 1. a PCA was performed to compare the miRNA expression profiles of the different sample groups. b Venn diagram of the differentially expressed miRNAs. It was observed that there were 13 miRNAs expressed in all groups.

Table 1. Expression fold changes of novel miRNAs

\begin{tabular}{|c|c|c|c|c|c|}
\hline Novel miRNA & Novel miRNA sequences & \multicolumn{4}{|c|}{ Fold changes compared to control } \\
\hline Novel-miR-2 & CCCGGCCGCGCCTGAGGGTT & 5.66 & 6.56 & 5.17 & 4.96 \\
\hline Novel-miR-3 & CGCCTGAGGGTTGCTTGCCC & 4.33 & 4.46 & 4.42 & 2.32 \\
\hline Novel-miR-4 & CGGACCCAGGAACTTCGCCCGA & 4.01 & 5.33 & 4.51 & 1.65 \\
\hline Novel-miR-7 & GCCCAGGAGATAGAGACC & 4.93 & 3.69 & 12.60 & 6.78 \\
\hline Novel-miR-8 & CGGGTGGGGGCGGGGCGG & 1.03 & 1.81 & 2.10 & 2.66 \\
\hline Novel-miR-9 & CGAGGGGGCGGGGCGGGGTC & 0.06 & 0.06 & 0.34 & 0.34 \\
\hline Novel-miR-10 & CGGCGGCGGTGGCGGCGGGA & 0.08 & 0.03 & 0.13 & 0.03 \\
\hline Novel-miR-11 & GGGACAGTGCCAGGTGGG & 0.06 & 0.06 & 0.15 & 0.15 \\
\hline
\end{tabular}

\section{Results}

\section{Global Statistics of Six Sequenced Small RNA}

Libraries of Novel miRNAs in PBMCs Infected with

EV71 and CA16

Our previous study comprehensively analyzed the known miRNAs; therefore, this study mainly focused on the analysis of novel miRNAs [16]. The background-subtracted signal intensities of the novel miRNAs were $\log _{2}$ transformed and quantile normalized. The normalized data were subsequently analyzed by PCA to determine if any intrinsic clustering or outliers existed within the data set. As shown in Figure 1a, the control group was clearly separated from the infected groups, whereas the EV71infected groups and the CA16-infected groups were grouped tightly and separately. This result implied that there was a remarkable difference between the EV71-infected and the CA16-infected groups. To understand the 


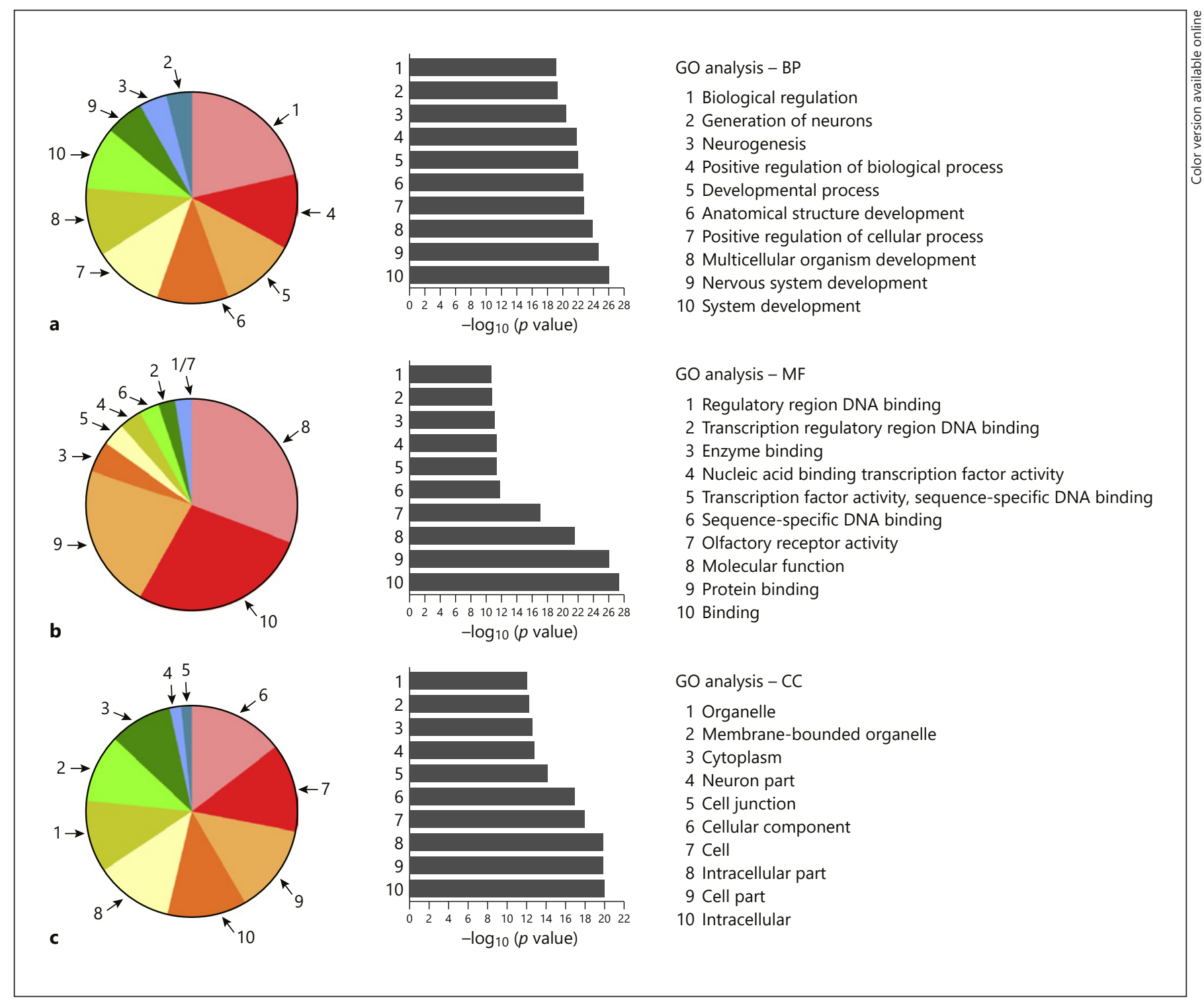

Fig. 2. The top 10 enriched GO terms were analyzed for the predicted target genes of the 13 novel miRNAs. a Enriched terms in the GO BP category. b Enriched terms in the GO CC category. c Enriched terms in the GO MF category. Bars indicate the $p$ value of the GO terms in the right panel. The vertical axis is the GO enrichment, and the horizontal axis is the negative logarithm of the $p$ value, which represents the significance level of the GO terms. effects of EV71 and CA16 infection on host novel miRNAs, we profiled the differential expression of novel miRNAs in response to EV71 and CA16 infection. It was found that there were only 13 novel differentially expressed miRNAs in the center of the Venn diagram, which indicated that these $13 \mathrm{miRNAs}$ overlapped among all four infected groups (Fig. 1b). The fold changes of these 13 miRNAs in four infected groups are shown in Table 1.

Novel microRNAs in PBMCs following EV71 and CA16 Infection
GO Annotation and KEGG Pathway Analysis of the

Predicted Target Genes of the Novel miRNAs

To further understand the roles of the aberrant miRNAs in physiological functions, the target genes of the 13 novel differentially expressed miRNAs were predicted. Using computational miRNA target gene prediction, we identified 3,781 putative targets with miRanda and 3,956 putative targets with RNAhybrid. Among these targets, 2,501 were predicted in both miRanda and RNAhybrid. Then, 

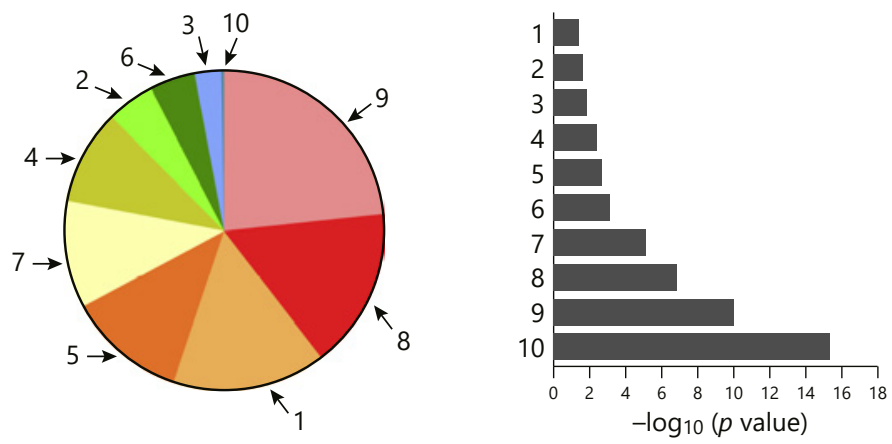

Pathway analysis

1 Transmembrane transport of small molecules

2 Cardiac conduction

3 lon homeostasis

4 GPCR downstream signaling

5 Signaling by NGF

6 Signaling by NOTCH

7 Neuronal system

8 Axon guidance

9 Developmental biology

10 Olfactory signaling pathway

Fig. 3. Pathway analysis based on the differentially expressed miRNA target genes. Pie chart of the top 10 enriched pathways. Bars indicate the $p$ value of the pathways in the right panel. The vertical axis is the pathway category, and the horizontal axis is the negative logarithm of the $p$ value, which represents the significance level of the pathways.

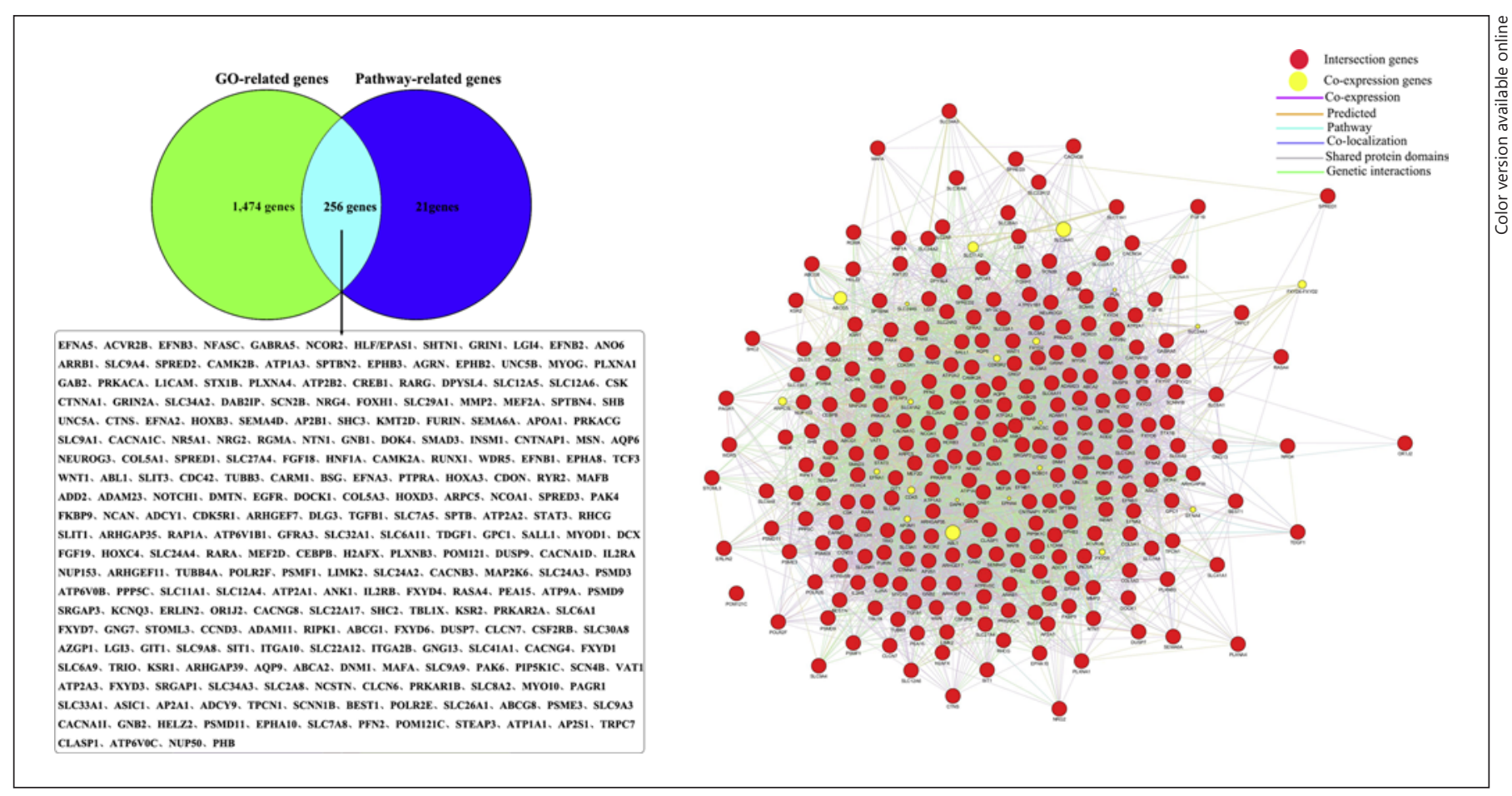

Fig. 4. a Map of the 256 overlapping target genes in the GO and KEGG pathway analyses. b Coexpression network constructed by GeneMANIA online software. The red nodes are the target genes, and the yellow nodes are coexpressed genes. Genes with larger nodes are more centralized in the network and have a stronger capacity for modulating adjacent genes. Different colored lines indicate different interactions between the genes.

the molecular functions of these intersectional targets were assessed by GO annotation and KEGG pathway analysis.

The biological significance of the target genes was established by performing an enrichment analysis with GO functions such as biological processes (BP), molecular functions (MF), and cellular components (CC). The results revealed that the 2,501 candidate target genes were distributed into $10 \mathrm{GO}$ terms based on BP (Fig. 2a), 10 GO terms based on MF (Fig. 2b), and 10 GO terms based on CC (Fig. 2c). 


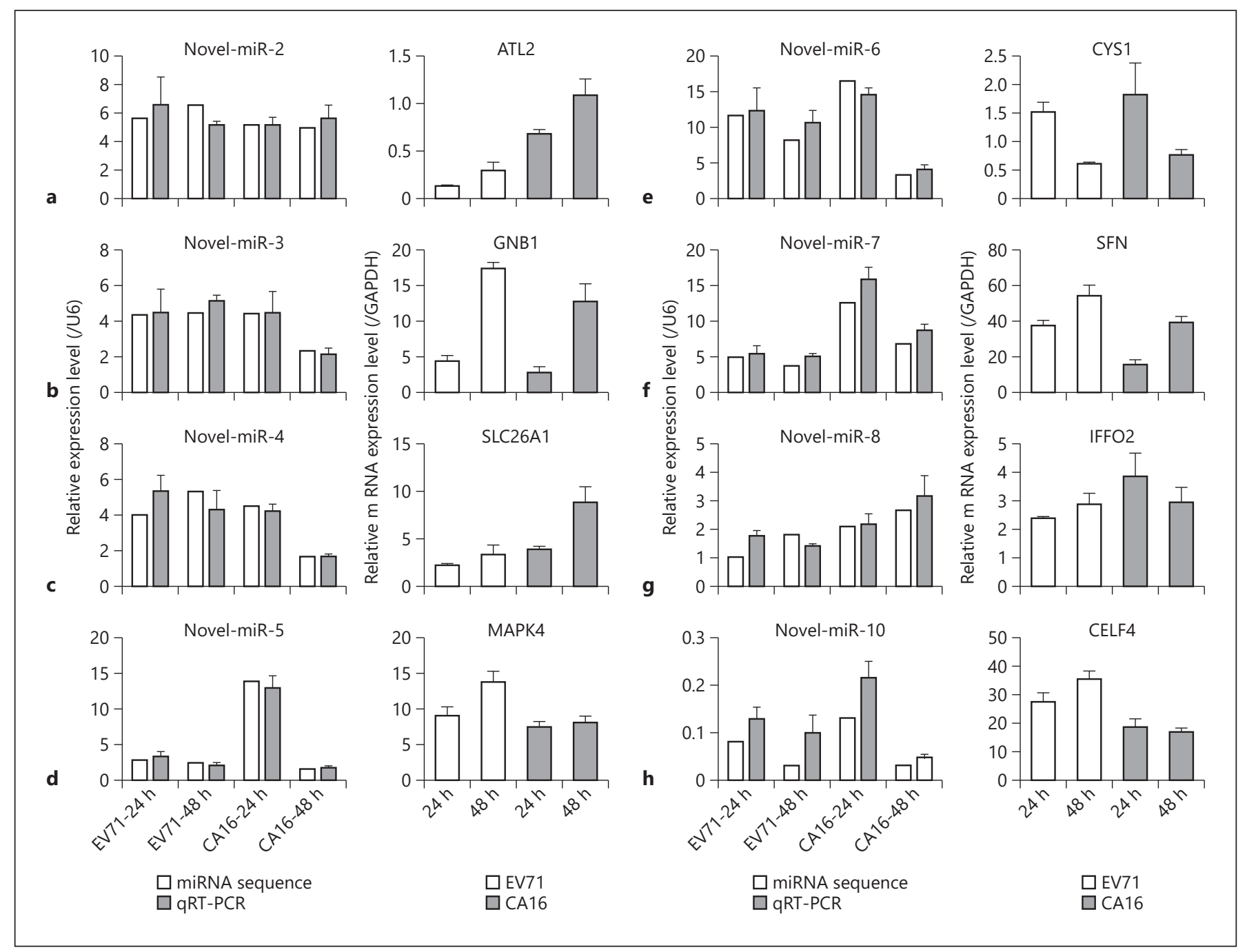

Fig. 5. Expression of novel miRNAs and their target genes was measured by RT-qPCR in PBMCs following EV71 and CA16 infection. a miR-2 and its target gene, ATL2. b miR-3 and its target gene, GNB1. c miR-4 and its target gene, SLC26A1. d miR-5 and its target gene, MARK4. e miR-6 and its target gene, CYS1. $\mathbf{f}$ miR-7 and its target gene, SFN. $\mathbf{g}$ miR-8 and its target gene, IFFO2. $\mathbf{h}$ miR-10 and its target gene, CELF4. The data are presented as the mean \pm SEM from three biological replicates.

KEGG pathway analysis can aid our understanding of the biological functions of target genes. In the KEGG enrichment analysis, olfactory signaling pathway, developmental biology, axon guidance, neuronal system signaling by NOTCH, signaling by NGF, GPCR downstream signaling, ion homeostasis, cardiac conduction, and transmembrane transport of small molecules were among the significantly enriched miRNA-associated pathways (Fig. 3).

\section{Coexpression Network of Target Genes}

Coexpression analysis is an important approach to explore the potential functions of genes. To understand the possible regulatory relationships of the key target genes, we performed coexpression network analysis using GeneMANIA online software. The key target genes were selected from the overlapped genes derived from the GO and KEGG pathway analyses (Fig. 4a); there were 256 genes in the intersectional section. Subsequently, these genes were used as input for the construction of a coexpression network (Fig. 4b). It was found that the coexpressed genes were closely associated with the key targets in many different ways, such as through genetic interactions, domains, and colocalization. 
Validation of miRNA and Target Gene Expression by $R T-q P C R$

RT-qPCR was performed to validate miRNA highthroughput sequencing results on 8 randomly selected miRNAs. The dynamic expression levels of the miRNAs in each group were consistent with the sequencing results (Fig. 5). Furthermore, we randomly selected 8 target genes to confirm the miRNA sequencing data. The results revealed that the expression levels of the target genes were inversely correlated with miRNA expression (Fig. 5).

\section{Discussion}

HFMD, which is mainly caused by EV71 and CA16, is a common childhood illness increasingly recognized as a significant health issue worldwide, particularly in the Asia-Pacific region [21]. Three inactivated EV71 vaccines have already been shown to provide substantial protection against EV71-HFMD and EV71-related diseases in clinical trials, but the current lack of an effective vaccine to treat HFMD induced by other enteroviruses, including CA16, highlights the importance of developing vaccines to prevent the transmission of the other causative agents [22]. Thus, further studies identifying the factors that cause the different manifestations of EV71 and CA16 infection are still urgently needed. Evidence has revealed the important function miRNAs play in viral disease, and there have been numerous reports of miRNAs that are connected to EV71 and CA16 infection. For example, Cui et al. [23] revealed that some miRNAs show differential expression levels following EV71 and CA16 infection, and three key miRNAs (miR-545, miR-324-3p, and miR-143) can be used to distinguish EV71 infection from CA16 infection in patients with HFMD. Furthermore, our previous studies have also demonstrated that there is significant differential expression of known miRNAs in EV71- and CA16-infected human cells $[1,15,16]$.

To elucidate the different molecular mechanisms underlying HFMD caused by EV71 and CA16 infection, we utilized EV71- and CA16-infected PBMCs of rhesus monkeys and miRNA high-throughput sequencing analysis to reveal the novel differentially expressed miRNAs in this study. It was found that 13 novel differentially expressed miRNAs were included in each group. These novel miRNAs were then used for clustering analysis, which showed that there was a clear difference between the infected samples and the control samples. In addition, the expression levels of the novel miRNAs were also examined, and the results suggested that there were distinc- tive miRNA expression patterns in PBMCs in response to EV71 and CA16 infection.

Target gene prediction for these novel differentially expressed miRNAs was carried out, and the results revealed 2,501 common targets with miRanda and RNAhybrid databases. Subsequently, the putative target genes of the novel miRNAs were analyzed by GO analysis in three categories: BP, MF, and CC. It was observed that a majority of the novel miRNA target genes were involved in the regulation of a broad range of metabolic and physiological processes; the enriched GO terms included nervous system-related terms (such as nervous system development, neurogenesis, generation of neurons, and neuron part), transcription-related terms (such as transcription regulatory region DNA binding, nucleic acid binding activity, transcription factor activity, and sequence-specific DNA binding), and adhesion-related terms (such as cell junction). However, it is well known that EV71 and CA16 are both highly neurotropic viruses that can result in severe neurological symptoms, including aseptic meningitis, brainstem encephalitis, and flaccid paralysis [24]. Thus, it was postulated that nervous system-related GO terms regulated by the novel differentially expressed miRNAs might be the key factors responsible for the inflammatory pathogenesis of the neurological symptoms. In addition, KEGG pathway analysis revealed that nervous system-related pathway annotations, such as those for neuronal systems and axon guidance, were significantly enriched.

The results further verified that these novel miRNAs can also mediate the function of the nervous system. Moreover, it was also discovered that signaling by Notch was remarkably abundant. Notch signaling plays critical roles during the differentiation and effector phases of innate and adaptive immune responses. Zhang et al. [25] reported that inhibition of Notch signaling combined with activation of Toll-like receptor signaling prevented formalin-inactivated respiratory syncytial virus (FIRSV) vaccine-enhanced respiratory disease, and the mechanism may involve suppressing proinflammatory Th17 memory responses and promoting protective tissue-resident memory in lungs. Additionally, Qin et al. [26] discovered that Notch signaling could modulate the balance of regulatory $\mathrm{T}$ cells and $\mathrm{T}$ helper 17 cells in patients with chronic HCV infection, and that the link between Notch signaling and Th cells might lead to a new intervention to break the immunotolerance of chronic $\mathrm{HCV}$ infection. Hence, it was speculated that the alteration of Notch signaling regulated by the novel differentially expressed miRNAs might be involved in modulat- 
ing immune responses in PBMCs subjected to EV71 and CA16 infection.

Finally, to gain further insights into the miRNA-regulated networks involved in EV71 and CA16 infection, the common target genes revealed by the GO and KEGG pathways analyses were used to create a coexpression regulatory network. Among all the coexpressed genes, ABL1 presented the largest node size and thus held an important position in this network. Increasing numbers of reports have demonstrated that ABL1 and related tyrosine kinases are involved in actin motility and affect the replication of certain viruses and bacteria by disrupting cytoskeletal organization $[27,28]$. Therefore, the key coexpressed gene ABL1 might play a significant role in the progression of EV71 and CA16 infection.

\section{Conclusion}

Our high-throughput sequencing data revealed novel miRNAs that were differentially expressed in EV71- and CA16-infected PBMCs of rhesus monkeys compared to control cells. In a previous study, we found that differentially expressed miRNAs are involved in the regulation of cell-cell junctions in 16HBE cells and HUVECs, but not in PBMCs $[1,16]$, whereas in PBMCs we found differentially expressed miRNAs associated with nervous system development and modulation of the immune response. This difference may be because 16HBE cells and HUVECs are epithelial cells and endothelial cells, respectively, and that cell-cell junctions play an important role in the barrier function of these cells. However, PBMCs are mainly a collection of immune cells, suggesting that neu- roimmune interactions perhaps contribute to the pathogenesis of EV71 and CA16 infections. It is worth noting that there are limitations to our study. Although the changes in the novel miRNA expression levels were detected based on the screening of samples from EV71- and CA16-infected PBMCs in vitro, whether they necessarily reflect the molecular pathogenesis of the virus in an in vivo setting remains to be elucidated. Therefore, further investigation is required to confirm our conclusions.

\section{Acknowledgements}

This study was supported by the CAMS Innovation Fund for Medical Sciences (2016-I2M-1-019 and 2017-I2M-2-006), the Youth Fund of the National Natural Science Foundation of China (31700153), the PUMC Youth Fund and the Fundamental Research Funds for the Central Universities (2017310039), Yunnan Applied Basic Research Projects (2018FB026), and 2017 Medical Reserve Talents of Yunnan Province (H-2017034).

\section{Statement of Ethics}

This study was approved by the Institutional Ethics Boards of the Institute of Medical Biology, Chinese Academy of Medical Sciences and Peking Union Medical College (approval No. YISHENGLUNZI [2013] 2) and conducted in accordance with the recommendations in the Guide for the Care and Use of Laboratory Animals of the Ministry of Science and Technology of the People's Republic of China.

\section{Disclosure Statement}

The authors declare that they have no competing interests.

\section{References}

1 Song J, Hu Y, Li J, Zheng H, Wang J, Guo L, et al. Different microRNA profiles reveal the diverse outcomes induced by EV71 and CA16 infection in human umbilical vein endothelial cells using high-throughput sequencing. PLoS One. 2017 May;12(5):e0177657.

2 Song J, Hu Y, Li J, Zheng H, Wang J, Guo L, et al. Suppression of the toll-like receptor 7-dependent type i interferon production pathway by autophagy resulting from enterovirus 71 and coxsackievirus a16 infections facilitates their replication. Arch Virol. 2018 Jan;163(1):135-44.

3 Yi EJ, Shin YJ, Kim JH, Kim TG, Chang SY. Enterovirus 71 infection and vaccines. Clin Exp Vaccine Res. 2017 Jan;6(1):4-14.
$4 \mathrm{Ng}$ Q, He F, Kwang J. Recent progress towards novel ev71 anti-therapeutics and vaccines. Viruses. 2015 Dec;7(12):6441-57.

5 Aswathyraj S, Arunkumar G, Alidjinou EK, Hober D. Hand, foot and mouth disease (HFMD): emerging epidemiology and the need for a vaccine strategy. Med Microbiol Immunol (Berl). 2016 Oct;205(5):397-407.

6 Li R, Liu L, Mo Z, Wang X, Xia J, Liang Z, et al. An inactivated enterovirus 71 vaccine in healthy children. N Engl J Med. 2014 Feb; 370(9):829-37.
7 Wang J, Qi S, Zhang X, Zhang Y, Liu L, Che $\mathrm{Y}$, et al. Coxsackievirus A 16 infection does not interfere with the specific immune response induced by an enterovirus 71 inactivated vaccine in rhesus monkeys. Vaccine. 2014 Jul;32(35):4436-42.

8 Mao Q, Wang Y, Yao X, Bian L, Wu X, Xu M, et al. Coxsackievirus A16: epidemiology, diagnosis, and vaccine. Hum Vaccin Immunother. 2014;10(2):360-7.

$9 \mathrm{Pu}$ J, Huang $\mathrm{H}$, Zhang $\mathrm{Y}$, Feng M, Yang E, Che $\mathrm{Y}$, et al. The structure, pathogenicity and immunogenicity of two virion fractions harvested from cell cultures infected with the ca16 virus. Intervirology. 2015;58(4):260-9. 
10 Ha M, Kim VN. Regulation of microRNA biogenesis. Nat Rev Mol Cell Biol. 2014 Aug; 15(8):509-24.

11 Trobaugh DW, Klimstra WB. Microrna regulation of rna virus replication and pathogenesis. Trends Mol Med. 2017 Jan;23(1):80-93.

12 Ono C, Fukuhara T, Motooka D, Nakamura S, Okuzaki D, Yamamoto S, et al. Characterization of miR-122-independent propagation of HCV. PLoS Pathog. 2017 May; 13(5):e1006374.

13 Song L, Liu H, Gao S, Jiang W, Huang W. Cellular microRNAs inhibit replication of the H1N1 influenza A virus in infected cells. J Virol. 2010 Sep;84(17):8849-60.

14 Wen BP, Dai HJ, Yang YH, Zhuang Y, Sheng R. MicroRNA-23b inhibits enterovirus 71 replication through downregulation of EV71 VPl protein. Intervirology. 2013;56(3):195200.

$15 \mathrm{Hu}$ Y, Song J, Liu L, Li J, Tang B, Zhang Y, et al. Comparison analysis of microRNAs in response to EV71 and CA16 infection in human bronchial epithelial cells by high-throughput sequencing to reveal differential infective mechanisms. Virus Res. 2017 Jan;228:90-101.

16 Hu Y, Song J, Liu L, Li J, Tang B, Wang J, et al. Different microRNA alterations contribute to diverse outcomes following EV71 and CA16 infections: insights from high-throughput sequencing in rhesus monkey peripheral blood mononuclear cells. Int J Biochem Cell Biol. 2016 Dec;81(Pt A):20-31.
17 Galili T, O’Callaghan A, Sidi J, Sievert C. Heatmaply: an R package for creating interactive cluster heatmaps for online publishing. Bioinformatics. 2018 May;34(9):1600-2.

18 Rehmsmeier M, Steffen P, Hochsmann M, Giegerich R. Fast and effective prediction of microRNA/target duplexes. RNA. 2004 Oct; 10(10):1507-17.

19 Betel D, Wilson M, Gabow A, Marks DS, Sander C. The microRNA.org resource: targets and expression. Nucleic Acids Res. 2008 Jan;36(Database issue):D149-53.

20 Huang W, Sherman BT, Lempicki RA. Systematic and integrative analysis of large gene lists using DAVID bioinformatics resources. Nat Protoc. 2009;4(1):44-57.

21 Lee KY. Enterovirus 71 infection and neurological complications. Korean J Pediatr. 2016 Oct;59(10):395-401.

22 Mao QY, Wang Y, Bian L, Xu M, Liang Z. EV71 vaccine, a new tool to control outbreaks of hand, foot and mouth disease (HFMD). Expert Rev Vaccines. 2016 May;15(5):599-606.

23 Cui L, Qi Y, Li H, Ge Y, Zhao K, Qi X, et al. Serum microRNA expression profile distinguishes enterovirus 71 and coxsackievirus 16 infections in patients with hand-foot-andmouth disease. PLoS One. 2011;6(11):e27071.
24 Solomon T, Lewthwaite P, Perera D, Cardosa MJ, McMinn P, Ooi MH. Virology, epidemiology, pathogenesis, and control of enterovirus 71. Lancet Infect Dis. 2010 Nov; 10(11): 778-90.

25 Zhang L, Li H, Hai Y, Yin W, Li W, Zheng B, et al. Cpg in combination with an inhibitor of notch signaling suppresses formalin-inactivated respiratory syncytial virus-enhanced airway hyperresponsiveness and inflammation by inhibiting th 17 memory responses and promoting tissue-resident memory cells in lungs. J Virol. 2017 Apr;91(10):91.

26 Qin L, Zhou YC, Wu HJ, Zhuo Y, Wang YP, $\mathrm{Si} \mathrm{CY}$, et al. Notch signaling modulates the balance of regulatory $t$ cells and thelper 17 cells in patients with chronic hepatitis c. DNA Cell Biol. 2017 Apr;36(4):311-20.

27 Hrincius ER, Liedmann S, Finkelstein D, Vogel P, Gansebom S, Ehrhardt C, et al. Nonstructural protein 1 (NS1)-mediated inhibition of c-Abl results in acute lung injury and priming for bacterial co-infections: insights into $1918 \mathrm{H} 1 \mathrm{~N} 1$ pandemic? J Infect Dis. 2015 May;211(9):1418-28.

28 García M, Cooper A, Shi W, Bornmann W, Carrion R, Kalman D, et al. Productive replication of Ebola virus is regulated by the cAbl1 tyrosine kinase. Sci Transl Med. 2012 Feb;4(123):123ra24. 